\title{
ANÁLISE DA EFICIÊNCIA DE RESFRIAMENTO DE PAINÉIS POROSOS PREENCHIDOS COM ARGILA EXPANDIDA EM COMPARAÇÃO AOS DE CELULOSE USANDO TÚNEL DE VENTO
}

José Francisco Vilela Rosa ${ }^{1}$, Ilda de Fátima Ferreira Tinôco², Carlos Magno Fernandes ${ }^{3}$, Sérgio Zolnier ${ }^{4}$, Mateus Marques Bueno 5

\section{RESUMO}

\begin{abstract}
Altas temperaturas do ar, associadass à tipologia aberta e sem isolamento térmico dos alojamentos para animais ainda constituem grave problema para seu desempenho produtivo. Neste sentido, os sistemas de acondicionamento de ambientes usuais nas atividades de produção animal brasileira geralmente fazem uso de processos de resfriamento adiabático evaporativo do ar, utilizando painéis de material poroso umedecido, associado aos sistemas de ventilação em modo túnel. Diante do exposto, este trabalho teve como objetivo comparar a eficiência de placas porosas em argila expandida (cinasita) em relação às do painel de celulose (testemunha) para todas as condições de temperaturas e velocidades das correntes de ar. Foram usados painéis em três espessuras $(6 \mathrm{~cm}, 8,5 \mathrm{~cm}$ e $10 \mathrm{~cm})$ e em duas granulometrias, denominadas de granulometria 1 (referência à brita 1) e granulometria 2 (referência à brita 2). Os resultados obtidos foram interpretados com testes de hipóteses de duas médias. Foram encontrados melhores valores de eficiência para os painéis em granulometria 1 em relação à granulometria 2. Também foi verificado bom desempenho para o painel evaporativo de $10 \mathrm{~cm}$ de espessura quando a granulometria da argila era a 2.
\end{abstract}

Palavras-chave: resfriamento evaporativo, painel evaporativo, eficiência de resfriamento, granulometria.

\section{ABSTRACT \\ COMPARATIVE EFFICIENCY ANALYSIS OF COOLING POROUS PADS OF EXPANDED CLAY AND CELLULOSE USING WIND TUNNEL}

High temperature associated with open typology and lack of thermal isolation of animal sheds seriously affects productive performance of animals. Generally, in Brazil the cooling systems are based on evaporative adiabatic cooling processes with humid porous material pads associated with ventilation systems in tunnel mode. This study compares the efficiency of porous pads made from expanded clay (cinasita) or from cellulose pads (control). Pads of 60,85 or $100 \mathrm{~mm}$ thickness, and two granulometries (grit 1 or grit 2) were used for all temperatures and airflow speeds. The results were analyzed according to hypothesis test of two means. The grit-1 pads were found to be most efficient. The $100 \mathrm{~mm}$ grit-2 pads also showed good performance.

Keywords: evaporative cooling, evaporative pads, cooling efficiency, granulometry.

\footnotetext{
Recebido para publicação em 29/05/2009. Aprovado em 28/05/2010.

1- Eng. Agrícola, Prof. Titular, D.S., Centro Univ. Newton Paiva, zevilela@terra.com.br

2- Enga . Agrícola, Prof ${ }^{a}$. Associado, Phd, UFV / DEA, iftinoco@ufv.br

3- Bacharel em Física, Prof. Adjunto. D.S. UFV/ DEA, cmagno@ufv.br

4- Eng. Agrícola, Prof. Associado, PhD, UFV / DEA, zolnier@ufv.br

5- Eng. Agrícola, UFV / DEA, mateusjuruaia@yahoo.com.br
}

\section{REVENG} 516-523 p. 


\section{INTRODUÇÃO}

A produção animal é o resultado do potencial genético das espécies. Conhecendo sua interação com a nutrição, sanidade, manejo e fatores ambientais, verifica-se que muitos animais não conseguem expressar todo o seu potencial produtivo sob as condições adversas do meio em que vivem. Assim, o ambiente é um dos responsáveis pelo sucesso ou fracasso dos empreendimentos, uma vez que pode ser definido como a soma dos impactos dos meios biológicos e físicos circundantes sobre os animais (CURTIS, 1983).

Nesse sentido, uma técnica de modificação ambiental artificial bastante difundida para o acondicionamento de ambientes é o resfriamento evaporativo do ar. De acordo com Carossi (2006) e Oliveira (2007), esse sistema apresenta vantagens sobre o sistema tradicional de condicionamento de ar, entre eles a redução no consumo de energia elétrica, o baixo custos de instalações e de manutenção e a umidificação e purificação pela filtragem do ar a ser insuflado e pela total renovação do ar ambiente, além de não ser poluente.

Em pesquisas anteriormente, foi demonstrado que a argila expandida (cinasita) tem características favoráveis de densidade, porosidade, durabilidade e disponibilidade no comércio, sugerindo tratar-se de material que satisfaz as exigências pertinentes a um bom material poroso, podendo substituir, com vantagem econômica, a celulose.

De acordo com Wiersma \& Stott (1974) e Bottcher et al. (1991), alguns países de clima seco e quente têm usado o sistema de resfriamento adiabático evaporativo, com economia e eficiência, para promover redução da temperatura em construções agrícolas. Contudo, o material usualmente empregado nesses equipamentos é uma placa de celulose, que pode vir acompanhada por uma série de inconvenientes, tais como custo elevado, baixa durabilidade, dificuldade de aquisição ou problemas com roedores, entre outros.

Os materiais comumente empregados nos enchimentos dos resfriadores evaporativos são a fibra de vidro, celulose impregnada com resina, polipropileno e fibra de madeira. O enchimento, além de promover a evaporação da água pelo ar, também atua como um filtro, retendo partículas acima de 3 micra (GLACIER-COR, 1999).

Tinôco et al. (2002) desenvolveram um estudo de comparação entre a eficiência de alguns materiais porosos alternativos encontrados no Brasil, entre eles a cinasita (argila expandida), a serragem, a fibra vegetal e o carvão, para o Sistema de Resfriamento Adiabático Evaporativo (SRAE). Foi avaliado o conforto térmico ambiente pelo Índice de Temperatura de Globo Negro e Umidade (ITGU) e Umidade Relativa do ar (UR). Concluiu-se que a confecção de placas porosas para resfriadores evaporativos, utilizando os materiais cinasita e carvão vegetal, indica vantagens sobre os demais materiais, e que, devido ao fato de a argila expandida (cinasita) ser um material facilmente encontrado no Brasil, ela poderia satisfazer as exigências de um bom material poroso, podendo substituir, com vantagem econômica, a celulose, dentro do processo de resfriamento evaporativo.

Vigoderis et al. (2007) desenvolveram uma pesquisa com o objetivo de construir um protótipo de túnel de vento que possibilitasse a avaliação da argila expandida como material poroso de placas para resfriamento evaporativo do ar, concluindo que a argila expandida é um material eficiente e apropriado para sistemas de resfriamento adiabático evaporativo, tendo o protótipo desenvolvido se mostrado apto para as análises.

Silva (2002) desenvolveu um trabalho com o objetivo de comparar o efeito dos painéis de celulose e de argila expandida no conforto térmico ambiente e no desempenho de frangos de corte, alojados em galpões avícolas comerciais climatizados, com sistema de ventilação negativa, em modo túnel, associados ao resfriamento adiabático evaporativo, tendo concluído que os painéis com argila expandida, embora tenham apresentado valores menores de eficiência evaporativa, têm potencial como material alternativo em resfriamento adiabático evaporativo.

No trabalho de Campos (2002), no qual têve-se como objetivo determinar o potencial de redução de temperatura do ar por meio de sistemas de resfriamento adiabático evaporativo para a região de Maringá-PR, utilizando dados climáticos da região, concluiu que os meses com maior potencial de redução de temperatura foram agosto, setembro, outubro e novembro, devido aos baixos valores de 
umidade relativa do ar. Os meses de dezembro, janeiro, fevereiro e março, apesar de se situarem no verão, apresentaram menor potencial de redução de temperatura por meio de resfriamento adiabático evaporativo devido à incidência de maiores valores de umidade relativa do ar. Entretanto, pelos valores analisados, conclui-se que esta técnica (resfriamento adiabático) é promissora para os horários mais críticos, das $13 \mathrm{~h}$ às $18 \mathrm{~h}$, com maior temperatura do ar.

Objetivou-se, com a realização do presente trabalho, comparar a eficiência de placas porosas em argila expandida (cinasita) em relação às do painel de celulose (testemunha) para todas as condições de temperaturas e velocidades das correntes de ar.

\section{MATERIAL E MÉTODOS}

O experimento foi realizado nos períodos de janeiro de 2007, abril de 2007 e janeiro de 2008, no Laboratório de Construções Rurais e Ambiência do Departamento de Engenharia Agrícola da Universidade Federal de Viçosa.

O túnel de vento utilizado nesta pesquisa foi construído e desenvolvido por Vigoderis (2002), com base em protótipo de Liao e Chiu (2002). Este equipamento permite testes de sistemas de resfriamentos evaporativos com painéis evaporativos fabricados por materiais alternativos (Figura 1).

O túnel de vento é composto pelas seguintes partes: câmara com 1,50 m de altura, 1,00 $\mathrm{m}$ de largura e 1,20 $\mathrm{m}$ de profundidade. Tais dimensões foram adotadas para que as características psicrométricas do ar pudessem ser avaliadas em no mínimo um metro de distância das placas porosas. O protótipo assim constituído tem uma abertura com dimensões de $1,23 \mathrm{~m}$ x 0,93 m para o encaixe dos painéis porosos. Uma redução metálica foi fixada numa de suas extremidades, convergindo para um duto circular. Faz parte do sistema um ventilador centrífugo de vazão de 1,6 $\mathrm{m}^{3} \mathrm{~s}^{-1}$, acionado por um motor de $5.500 \mathrm{~W}, 220 / 380$ Volts, trifásico, de $1750 \mathrm{rpm}$. A extremidade livre do ventilador tem um anteparo regulável com a função de variar a vazão do ar (Vigoderis, 2002).

Durante os testes, os painéis de material poroso eram aspergidos com água para permitir o molhamento da celulose corrugada e da argila expandida, promovendo a evaporação e consequente resfriamento com a passagem do ar. O sistema de molhamento por lâmina de água foi constituído de um tubo de PVC, posicionado logo acima do painel, perfurado num total de 28 furos, onde eram formados jatos de água com uma lâmina homogênea que escoava pelo material poroso ( Figura 2). Este tubo era abastecido pela água de uma caixa d'água abaixo dos painéis evaporativos, na qual foi colocada uma calha metálica para a coleta da água excedente, que deixava o material poroso.

Foram utilizados três painéis com dimensões fixas, com espessuras de $6,0,8,5 \mathrm{~cm}$ e $10,0 \mathrm{~cm}$, e, como testemunha, placas de celulose da marca "Munters ${ }^{\circledR} "$ com proteção de tinta betuminosa na face externa, com dimensões de $1,23 \times 0,31 \times 0,15$ m (Figura 3).

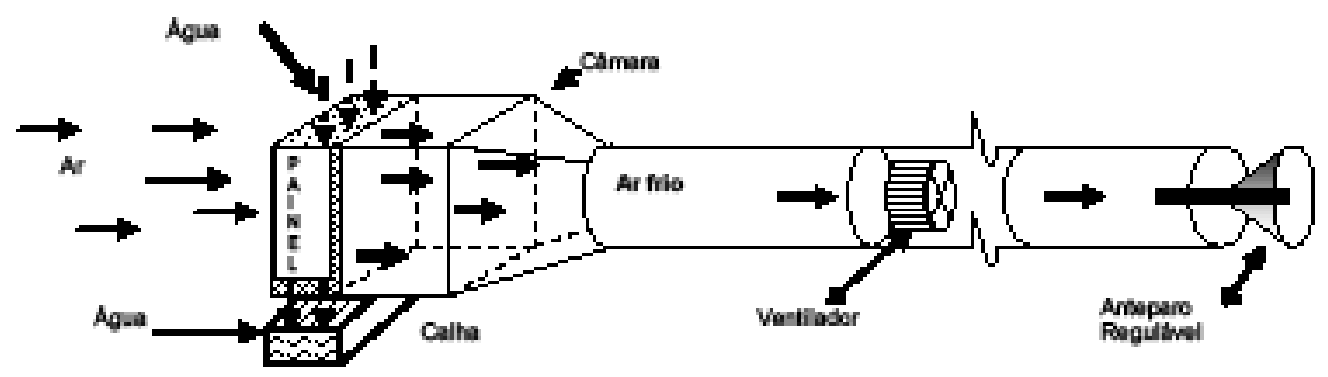

Fonte: $\mathrm{O}$ autor.

Figura 1. Representação esquemática do túnel e do painel de resfriamento evaporativo. 


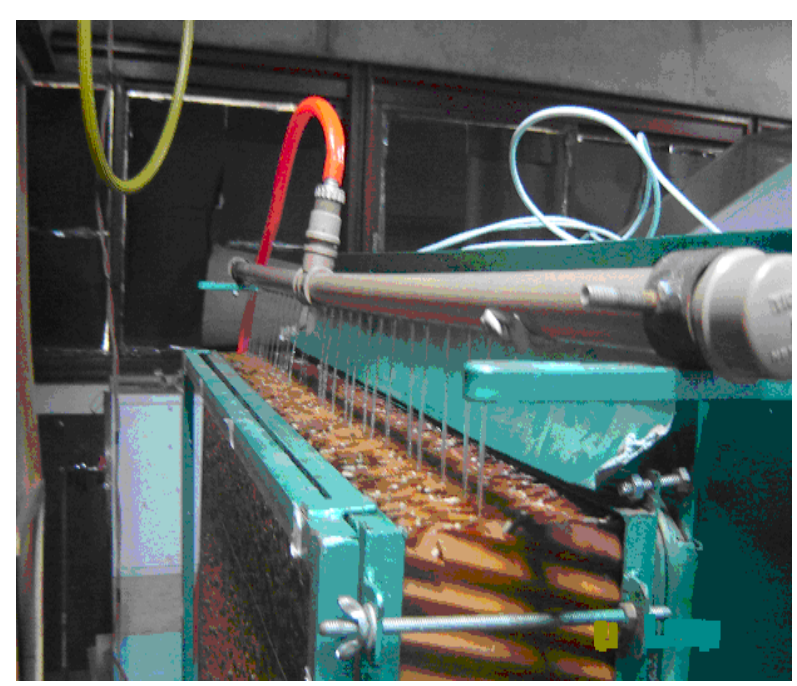

Figura 2. Água borrifando sobre o painel.

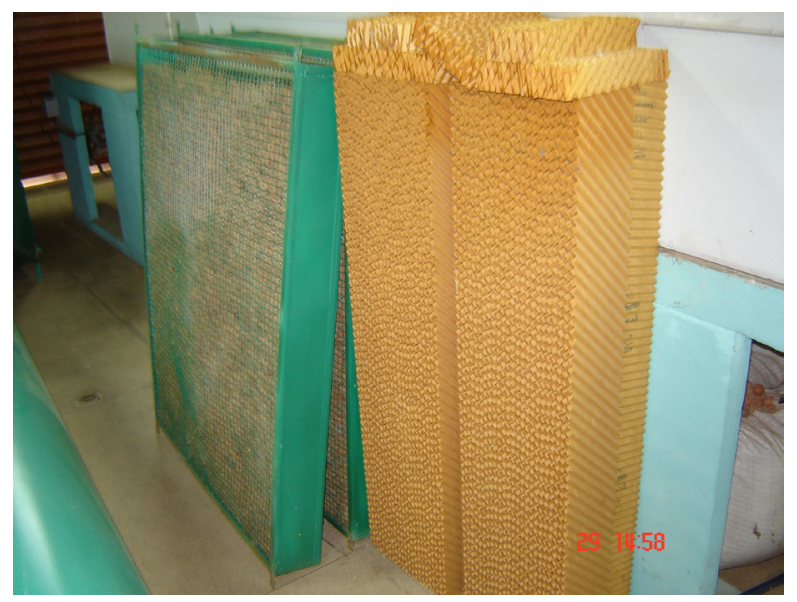

Figura 3. Painéis com argila expandida e placas de celulose usadas como testemunha.

Em intervalos de 30 segundos foram medidos valores de temperatura de bulbo seco do ar externo e do ar interior, umidade relativa do ar externo, velocidade do ar próximo aos painéis evaporativos e pressão estática para quatro diferentes aberturas do regulador de vazão.

A temperatura e a umidade relativa do ar foram medidas por meio de um instrumento que combina dois sensores independentes: um para a temperatura e outro para a umidade relativa do ar (Modelo HUM50Y, VAISALA, Woburn, MA). Além desses sensores, termopares do tipo " $\mathrm{T}$ " foram instalados no interior do túnel para leituras das temperaturas. As velocidades do ar foram medidas posicionando o aparelho medidor no centro geométrico de 20 pontos simetricamente separados próximos do painel, determinando-se a velocidade média do ar por repetição antes de sua passagem pela placa porosa. Para essas leituras, foi utilizado um anemômetro de fio quente (Modelo FMA-903-I, OMEGA, Stanford). As leituras de pressão estática foram obtidas utilizando-se um Micromanômetro do fabricante KIMO, modelo MP 120, com medidas em $\mathrm{mmH}_{2} 0$ e acurácia de \pm $2 \mathrm{mmH}_{2} 0$, posicionado na lateral do túnel.

Os dados relativos ao ambiente externo próximo ao painel e também no interior do túnel foram obtidos por meio de um sistema automático de aquisição de dados, tendo como plataforma um microcomputador. Nessa configuração, uma placa de aquisição de dados (CYDAS 1620HR, CYBERRESEARCH, Branford, CT) de 16 canais de entrada analógica com 16 bits foi instalada em um barramento ISA do microcomputador. Além da placa principal, foi utilizada uma placa de expansão CYEXP32 para amplificação dos sinais provenientes dos termopares tipo T. Os canais analógicos foram conectados aos sensores responsáveis pelo monitoramento do ambiente físico.

Para a realização dos testes experimentais, fezse a interação entre três fatores influenciadores no processo de resfriamento adiabático evaporativo: tipo de argila expandida, espessura do painel e vazão do ar.

Foram utilizadas duas granulometrias de argila expandida (cinasita) equivalentes à Brita 1 e Brita 2, denominadas Granulometria $1\left(\mathrm{G}_{1}\right)$ e Granulometria $2\left(\mathrm{G}_{2}\right)$, respectivamente.

As espessuras dos painéis evaporativos utilizadas na avaliação foram de $6 \mathrm{~cm}\left(\mathrm{E}_{1}\right), 8,5 \mathrm{~cm}$ $\left(\mathrm{E}_{2}\right)$ e $10 \mathrm{~cm}\left(\mathrm{E}_{3}\right)$. Cada abertura do regulador de vazão foi denominada $V_{1}, V_{2}, V_{3}$ e $V_{4}$, sendo $V_{1}$ a menor e $V_{4}$ a maior vazão de ar. Dessa forma, com duas granulometrias, três espessuras e quatro vazões, formaram-se 24 conjuntos de medidas para os testes; e, para cada conjunto foram feitas três séries de repetições.

A eficiência do resfriamento adiabático evaporativo $(\varepsilon)$ foi calculada por meio da equação (CASTRO e PIMENTA, 2004):

$\varepsilon=\frac{T_{\text {ext }}-T_{\text {int }}}{T_{\text {ext }}-T_{\text {BU }}} 100 \%$

em que 
$\mathrm{T}_{\text {ext }}=$ temperatura de bulbo seco do ar, exterior $\left({ }^{\circ} \mathrm{C}\right)$; $\mathrm{T}_{\text {int }}=$ temperatura de bulbo seco do ar, interior $\left({ }^{\circ} \mathrm{C}\right)$; e $\mathrm{T}_{\mathrm{BU}}=$ temperatura de bulbo molhado do ar, antes de ultrapassar o painel $\left({ }^{\circ} \mathrm{C}\right)$.

A determinação da temperatura de bulbo $\left(\mathrm{T}_{\mathrm{BU}}\right)$ molhado foi obtida por meio do método numérico de Newton, utilizando os valores psicrométricos medidos. Para comparar as eficiências, os dados obtidos foram interpretados com testes de hipóteses de duas médias, partindo-se da hipótese de que as eficiências médias desses dois materiais sejam iguais. Como hipótese alternativa, admitiu-se que as médias entre esses materiais eram diferentes. Para comparar as médias das eficiências entre os painéis, usou-se o teste " $t$ " de Student, em nível de significância de 5\%.

\section{RESULTADOS E DISCUSSÃO}

No Quadro 1 mostram-se os resultados, relacionando as espessuras, a abertura do regulador de vazão, as médias de eficiências de cinasita e de celulose.

Analisando os dados apresentados no Quadro 1, pode-se observar que as eficiências médias para a primeira abertura do regulador de vazão (menor velocidade do ar estudada para este caso), argila expandida com granulometria 1 e três espessuras analisadas não apresentaram diferença estatística com relação aos valores de eficiência determinados para a celulose corrugada. Já para os painéis preenchidos com argila expandida com granulometria 2, tal comportamento foi detectado apenas para o painel de espessura de $10 \mathrm{~cm}$. Nas segunda, terceira e quarta

Quadro 1. Pressão estática, vazão de ar úmido. Valores médios de eficiência dos painéis de cinasita e de celulose por abertura do regulador de vazão e espessuras, valores de t calculados pelo teste $t$ de Student.

\begin{tabular}{|c|c|c|c|c|c|c|c|}
\hline Granulometria & $\begin{array}{l}\text { Espessura } \\
\quad(\mathrm{cm})\end{array}$ & Abertura & $\underset{(\mathrm{kg} / \mathrm{s})}{\dot{m}}$ & $\mathrm{Pe}(\mathrm{Pa})$ & $\begin{array}{l}\text { Média } \\
\text { cinasita }\end{array}$ & $\begin{array}{c}\text { Média } \\
\text { Celulose }\end{array}$ & $\mathrm{t}_{\text {calc }}$ \\
\hline \multirow{12}{*}{1} & $6,0 \mathrm{~cm}$ & \multirow{3}{*}{ V1 } & 0,92 & 76,2 & 75 & 74,7 & 0,09 \\
\hline & $8,5 \mathrm{~cm}$ & & 0,54 & 94,8 & 69,9 & 74,7 & $-1,38$ \\
\hline & $10,0 \mathrm{~cm}$ & & 0,78 & 101,1 & 73 & 74,7 & $-0,39$ \\
\hline & $6,0 \mathrm{~cm}$ & \multirow{3}{*}{$\mathrm{V} 2$} & 1,49 & 91,61 & 66,6 & 79 & $-6,89$ \\
\hline & $8,5 \mathrm{~cm}$ & & 0,92 & 119,9 & 89,3 & 79 & 2,38 \\
\hline & $10,0 \mathrm{~cm}$ & & 1,21 & 134,8 & 67,1 & 79 & $-1,47$ \\
\hline & $6,0 \mathrm{~cm}$ & \multirow{3}{*}{ V3 } & 1,64 & 96,6 & 67,4 & 78,9 & $-8,29$ \\
\hline & $8,5 \mathrm{~cm}$ & & 1,06 & 129,9 & 76,5 & 78,9 & $-0,92$ \\
\hline & $10,0 \mathrm{~cm}$ & & 1,42 & 148,7 & 72,2 & 78,9 & $-3,70$ \\
\hline & $6,0 \mathrm{~cm}$ & \multirow{3}{*}{ V4 } & 1,67 & 110,7 & 64 & 79,7 & $-5,12$ \\
\hline & $8,5 \mathrm{~cm}$ & & 1,09 & 137,4 & 77,5 & 79,7 & $-1,00$ \\
\hline & $10,0 \mathrm{~cm}$ & & 1,85 & 156,4 & 69,7 & 79,7 & $-3,98$ \\
\hline \multirow{12}{*}{2} & $6,0 \mathrm{~cm}$ & \multirow{3}{*}{ V1 } & 0,87 & 67,9 & 42 & 74,7 & $-9,60$ \\
\hline & $8,5 \mathrm{~cm}$ & & 0,84 & 86,2 & 50,7 & 74,7 & $-8,42$ \\
\hline & $10,0 \mathrm{~cm}$ & & 0,84 & 86,2 & 74,7 & 74,7 & 0 \\
\hline & $6,0 \mathrm{~cm}$ & \multirow{3}{*}{ V2 } & 1,31 & 83,3 & 50,1 & 79 & $-17,31$ \\
\hline & $8,5 \mathrm{~cm}$ & & 1,39 & 109,0 & 48,3 & 79 & $-9,78$ \\
\hline & $10,0 \mathrm{~cm}$ & & 1,39 & 109,0 & 78,8 & 79 & $-0,07$ \\
\hline & $6,0 \mathrm{~cm}$ & \multirow{3}{*}{ V3 } & 1,70 & 87,8 & 52,6 & 78,9 & $-13,18$ \\
\hline & $8,5 \mathrm{~cm}$ & & 1,48 & 118,0 & 53,5 & 78,9 & $-9,396$ \\
\hline & $10,0 \mathrm{~cm}$ & & 1,49 & 118,0 & 79,1 & 78,9 & 0,09 \\
\hline & $6,0 \mathrm{~cm}$ & \multirow{3}{*}{ V4 } & 1,75 & 100,7 & 49,9 & 79,7 & $-5,99$ \\
\hline & $8,5 \mathrm{~cm}$ & & 1,67 & 125,0 & 53,8 & 79,7 & $-13,35$ \\
\hline & $10,0 \mathrm{~cm}$ & & 1,68 & 125,0 & 80,1 & 79,7 & 0,27 \\
\hline
\end{tabular}


aberturas, os resultados foram similares, isto é, os painéis de cinasita com granulometria 1 apresentaram evidências de que as eficiências médias são iguais aos do painel de celulose para as espessuras de 8,5 $\mathrm{cm}$ e $10,0 \mathrm{~cm}$; já para os painéis com argila com granulometria 2, apenas com a espessura de $10 \mathrm{~cm}$ foi igual.

Os resultados médios das eficiências dos sistemas de resfriamento adiabáticos evaporativos alcançados por cada conjunto de fatores como granulometria (Granulometria 1 - G1 e Granulometria 2 - G2) e espessuras $(6 \mathrm{~cm}, 8,5 \mathrm{~cm}$ e $10 \mathrm{~cm})$, por abertura do regulador de vazão, são mostrados na Figura 4.

Verifica-se pela Figura 4 que, para todos os valores de vazão do ar com a granulometria 2, o tratamento que apresentou valores de eficiência de resfriamento mais próximos aos obtidos na testemunha (painel de celulose) foi o de $10 \mathrm{~cm}$.

Verifica-se, também, que para os conjuntos " $6 \mathrm{~cm}$ G1" e "10 cm G1" foram obtidas eficiências em torno de $70 \%$, sendo que para o painel de espessura " $8,5 \mathrm{~cm}$ G1" as eficiências alcançadas foram um pouco maiores, sugerindo que essa espessura intermediária do painel poroso indica ser a que possibilita melhor eficiência de resfriamento do sistema. Este resultado possivelmente ocorre em razão de a espessura intermediária possibilitar um maior tempo de contato entre o ar não saturado a ser resfriado e a água presente no painel, intensificando a evaporação da água e o umedecimento do ar. Já para o painel de maior espessura $(10,0 \mathrm{~cm})$, a intensidade de evaporação pode ser reduzida em razão da maior obstrução à passagem do ar. Assim, embora o tempo de contato do ar e água possa ser maior, o volume de ar a ser resfriado se reduz, afetando negativamente a eficiência do sistema, de acordo com Tinôco (2002) e Vigoderis (2002).

$\mathrm{O}$ experimento com argila expandida na granulometria 2, (mais grossa), no entanto, apresentou resultados diferentes. Neste caso, o painel com espessura maior $(10 \mathrm{~cm})$ em média apresentou valores de eficiência de resfriamento maiores que aqueles obtidos em painéis de 6 e $8,5 \mathrm{~cm}$ de espessura. Neste caso, infere-se que sendo a granulometria maior, maior também será a porosidade, facilitando a passagem do ar quando comparado com as granulometrias menores. Sendo assim, para resguardar um maior contato do ar com a água, torna-se necessária uma maior espessura do painel evaporativo.

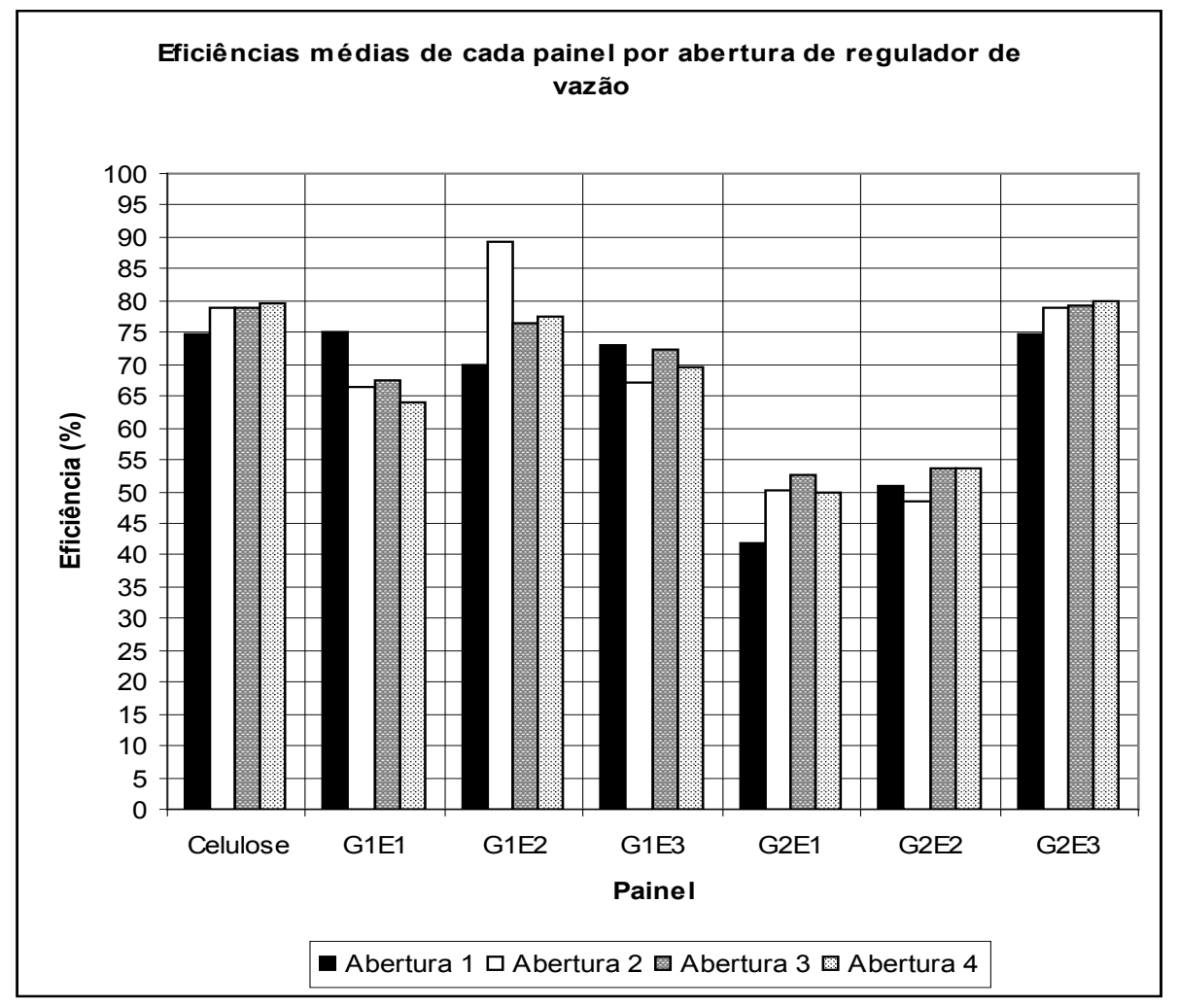

Figura 4. Valores de eficiência em função das características de cada painel. 
Comparativamente, o ensaio realizado com painéis de argila expandida de Granulometria 1, nas espessuras 6 e $8,5 \mathrm{~cm}$, apresentou valores de eficiência evaporativa entre 70 e $80 \%$, considerados melhores que aqueles obtidos na Granulometria 2 , de cerca de $50 \%$, para as mesmas espessuras de painéis.

Subentende-se que, para espessuras de painéis inferiores a $8,5 \mathrm{~cm}$, granulometrias menores conferem maior eficiência de resfriamento por reterem o ar por mais tempo no painel devido à maior resistência à sua passagem. Este fato possibilitaria maior eficiência no sistema adiabático de troca de calor e massa entre ar e água e consequente resfriamento da temperatura de ambos. Estes resultados estão de acordo com Wiersma e Stott (1983).

Todos estes resultados demonstram que a eficiência de arrefecimento térmico promovida pelos sistemas de resfriamento adiabático evaporativo do ar depende da granulometria da argila expandida, assim como da vazão do ar.

\section{CONCLUSÕES}

- Foram encontrados melhores valores de eficiência de resfriamento para os painéis confeccionados com argila expandida na granulometria 1 em relação à granulometria 2 para todas as espessuras de painéis adotadas;

- Todos os painéis evaporativos confeccionados em argila expandida de granulometria 1, nas espessuras $6,0 \mathrm{~cm}, 8,5 \mathrm{~cm}$ e 10,0 $\mathrm{cm}$, apresentaram resultados de eficiência de resfriamento adiabático evaporativo compatíveis com os obtidos pelo painel de celulose; e

- Os painéis de argila expandida com granulometria 2 apresentaram melhores eficiências de resfriamento para o painel evaporativo de espessura de $10 \mathrm{~cm}$.

\section{REFERÊNCIAS BIBLIOGRÁFICAS}

BOTTCHER, R.W.; BAUGHMAN, G. R.; GATES, R. S. Characterizing efficiency of misting systems for poultry. Transactions of the ASAE, St. Joseph, v.34, n.2, p.586-590, 1991.

CAMPOS,A.T.; KLOSOWSKI, E.S.; GASPARINO, E.; CAMPOS, A.T. Estudo do potencial de redução da temperatura do ar por meio do sistema de resfriamento adiabático evaporativo na região de Maringá, Estado do Paraná. Acta Scientiarum. Maringá, v. 24, n. 5, p. 1575-1581, 2002.

CAROSSI, G.A.B. Resfriamento evaporativo: Estudo do potencial de sua aplicação no Brasil. Uberlândia: UFU, 2006, 110p. Tese de mestrado.

CASTRO, W.P.; PIMENTA, J.M.D. Modelagem e simulação de painéis evaporativos diretos. In: CONGRESSO BRASILEIRO DE ENGENHARIA E CIÊNCIAS TÉRMICAS, 10. Anais... Rio de Janeiro - RJ, 2004.

CURTIS, S.E. Environmental management in animal agriculture. Ames: The Iowa State University Press, 409 p., 1983. 409p.

GLACIER-COR CELLULOSE EVAPORATIVE COOLING PADS. Operation and maintenance manual. Scottsdale, Arizona, 1999. (Catálogo do fabricante)

LIAO, C.M.; CHIU, K.H. Wind tunnel modeling the system performance of alternative evaporative cooling pads in Taiwan region. Building and Environment, v.37, p.177-187, 2002.

OLIVEIRA, A.M. Climatização por resfriamento evaporativo: estudo teórico e experimental de protótipos. Belo Horizonte: UFMG, 2007, 102p; Tese de Doutorado.

SILVA, C.E. Comparação de painéis evaporativos de argila expandida e celulose para sistema de resfriamento adiabático do ar em galpões avícolas com pressão negativa em modo de túnel. Viçosa: UFV, 2002, 67p. Tese de Mestrado.

TINÔCO, I.F.F.; FIGUEIREDO, J.L.A.; SANTOS, R.C.; PAULA, M.O.; PUGLLESI, N. L.; VIGODERIS. R, B. Avaliação de materiais alternativos utilizados na confecção de placas 
porosas para sistemas de resfriamento adiabático evaporativo. Revista Brasileira de Engenharia Agrícola e Ambiental, Campina Grande, v.6, n.1, p.147-150, 2002.

VIGODERIS, R.B. Desenvolvimento de um protótipo para resfriamento adiabático evaporativo, em instalações climatizadas para animais, usando argila expandida. 2002. Dissertação (Mestrado em Engenharia Agrícola) Universidade Federal de Viçosa, Viçosa - MG, 2002.

VIGODERIS, R.B.; TINÔCO, I.F.F.; LACERDA
FILHO, A.F.; SILVA, J.N., GATES, R.S., PAULI, D.G.; SILVA, C.E. \& GUIMARÃES, M.C.C. Construção de túnel de vento reduzido, visando à avaliação de argila expandida em sistemas de resfriamento adiabático evaporativo para arrefecimento térmico em galpões de produção animal. Engenharia na Agricultura, Viçosa, MG, v. 15, n.3, p.191-199, 2007.

WIERSMA, F.; STOTT, F.M. Response of dairy cattle to an evaporative cooled environment. Proceedings of the Livestock Environment. St. Joseph: American Society of Agricultural Engineers. 1974. p.88-95. 